\title{
APPARENT CONTOURS WITH MINIMAL NUMBER OF SINGULARITIES
}

\author{
Takahiro YAMAMOTO \\ (Received 24 March 2008 and revised 20 July 2009)
}

\begin{abstract}
For a stable map $\varphi: M \rightarrow S^{2}$ of a closed and connected surface into the sphere, let $c(\varphi), i(\varphi)$ and $n(\varphi)$ denote the numbers of cusps, fold curve components and nodes respectively. In this paper, in a given homotopy class, we will determine the minimal triples $(c, i, n),(n, c, i)$ and $(i, n, c)$ with respect to the lexicographic order.
\end{abstract}

\section{Introduction}

Let $M$ be a closed and connected surface and $N$ a connected surface. Let $\varphi: M \rightarrow N$ be a $C^{\infty}$ map. Define the set of singular points of $\varphi$ as

$$
S(\varphi)=\left\{p \in M \mid \operatorname{rank} d \varphi_{p}<2\right\} .
$$

We call $\varphi(S(\varphi))$ the apparent contour (or contour for short) of $\varphi$ and denote it by $\gamma(\varphi)$. When $S(\varphi) \subset M$ consists of one component, $\gamma(\varphi)$ is said to be irreducible.

A $C^{\infty} \operatorname{map} \varphi: M \rightarrow N$ is said to be stable if it satisfies the following two properties.

(1) The map germ at each $p \in M$ is $C^{\infty}$ right-left equivalent to one of the map germs at $0 \in \mathbb{R}^{2}$ below:

- $(a, x) \mapsto(a, x): p$ is a regular point;

- $(a, x) \mapsto\left(a, x^{2}\right): p$ is a fold point;

- $(a, x) \mapsto\left(a, x^{3}+a x\right): p$ is a cusp point.

Hence, $S(\varphi)$ is a finite disjoint union of circles.

(2) For each $q \in \gamma(\varphi)$, the map germ $\left(\left.\varphi\right|_{S(\varphi)}, \varphi^{-1}(q) \cap S(\varphi)\right)$ is right-left equivalent to one of the three multi-germs as depicted in Figure 1.

According to a classical result of Whitney [10], stable maps form an open everywhere dense set in the space of all $C^{\infty}$ maps $M \rightarrow N$. Thus, for each homotopy class of a $C^{\infty}$ map $M \rightarrow N$, there is a stable map.

In this paper, we consider stable maps with singular points.

When $\varphi$ is stable, $S(\varphi)$ is called the fold curve of $\varphi$, and the numbers of cusps, fold curve components and nodes on $\gamma(\varphi)$ are denoted by $c(\varphi), i(\varphi)$ and $n(\varphi)$ respectively. A stable map that has no cusp is called a fold map.

2000 Mathematics Subject Classification: Primary 57R45; Secondary 57N13.

Keywords: stable map; cusp; node; genus; mapping degree.

(C) 2010 Faculty of Mathematics, Kyushu University 


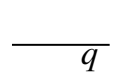

Fold

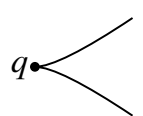

Cusp

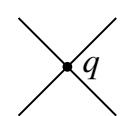

Node

FIGURE 1. The multi-germs of $\left.\varphi\right|_{S(\varphi)}$.

An oriented closed surface (or a non-orientable closed surface) of genus $g$, that is a connected sum of $g$ copies of the two-dimensional torus $T^{2}$ (respectively $g$ copies of the real projective plane $\mathbb{R} P^{2}$ ), is denoted by $\Sigma_{g}$ (respectively $F_{g}$ ). The two-dimensional sphere and the plane are denoted by $S^{2}$ and $\mathbb{R}^{2}$ respectively.

The apparent contours of stable maps $M \rightarrow \mathbb{R}^{2}$ or $S^{2}$ have been studied by some authors $[\mathbf{1}, \mathbf{2}, \mathbf{4}, \mathbf{5}]$. Recall the following proposition in [2].

Proposition 1.1. [2] Let $f: \Sigma_{g} \rightarrow S^{2}$ be a degree $d$ stable map whose contour is irreducible. Then:

(1) the contour $\gamma(f)$ has at least two cusps if $|d| \leq g$ and $d \not \equiv g(\bmod 2)$;

(2) the contour $\gamma(f)$ has at least $2(|d|-g)$ cusps if $|d| \geq g$.

Proposition 1.1 shows that there is a relation among the number of fold curve components and the number of cusps for a stable map between surfaces.

We recall that, for each pair $(d, g)$ satisfying $|d| \leq g$ and $d \equiv g(\bmod 2)$, a degree $d$ fold map $\Sigma_{g} \rightarrow S^{2}$ whose contour is irreducible was obtained in [2].

We remark that, for a stable map $\varphi: M \rightarrow S^{2}$, the number of cusps of $\varphi$ and the Euler characteristic of $M$ have the same parity by a classical result of Thom [7].

In this paper, for a given $C^{\infty}$ map $\varphi_{0}: M \rightarrow N$, we study three kinds of apparent contours of stable maps that are homotopic to $\varphi_{0}$. One is a $(c, i, n)$-minimal contour of $\varphi_{0}$. The others are an $(n, c, i)$-minimal contour of $\varphi_{0}$ and an $(i, n, c)$-minimal contour of $\varphi_{0}$.

Let $\varphi_{0}: M \rightarrow N$ be a $C^{\infty}$ map and $\varphi: M \rightarrow N$ be a stable map. The contour $\gamma(\varphi)$ is called a $(c, i, n)$-minimal contour, an $(n, c, i)$-minimal contour and an $(i, n, c)$-minimal contour of $\varphi_{0}$ if the triples $(c(\varphi), i(\varphi), n(\varphi)),(n(\varphi), c(\varphi), i(\varphi))$ and $(i(\varphi), n(\varphi), c(\varphi))$ are the smallest with respect to the lexicographic order among the stable maps that are homotopic to $\varphi_{0}$ respectively.

We recall that by virtue of Hopf's theorem (for example, see [3]), two $C^{\infty}$ maps $\Sigma_{g} \rightarrow S^{2}$ (or $F_{g} \rightarrow S^{2}$ ) are homotopic if and only if their degrees (respectively modulo two degrees) coincide. Thus, the homotopy classes of a degree $d$ stable map $\Sigma_{g} \rightarrow S^{2}$ and a modulo two degree $d_{2}$ stable map $F_{g} \rightarrow S^{2}$ are determined by the pairs $(d, g)$ and $\left(d_{2}, g\right)$ respectively.

The following theorem concerning a $(c, i, n)$-minimal contour is the first result of this paper. 

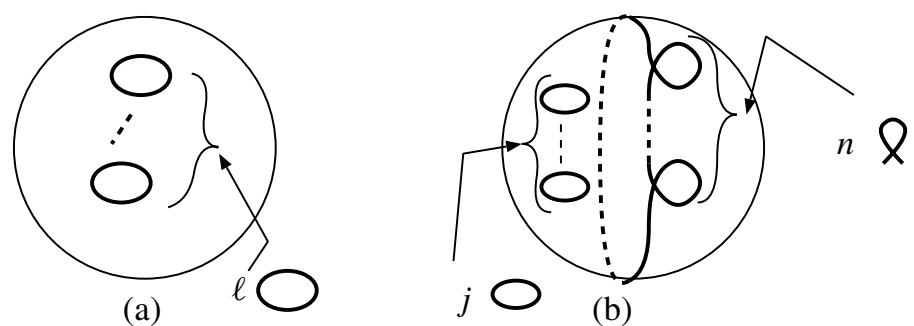

FiguRE 2. The $(c, i, n)$-minimal contours of $C^{\infty}$ map $\Sigma_{g} \rightarrow S^{2}$.

\section{THEOREM 1.2.}

(1) Let $f: \Sigma_{g} \rightarrow S^{2}$ be a stable map of degree $d$. Then, $\gamma(f)$ is $(c, i, n)$-minimal if and only if the triple $(c, i, n)$ for $\gamma(f)$ is one of the items below:

$$
(c, i, n)=\left\{\begin{array}{l}
\text { (i) }(0,|d|+1,0) \\
\quad \text { if } g=0 ; \\
\text { (ii) }(0,2,0) \\
\text { if }(|d|, g)=(0,1) \\
\text { (iii) }(0,1,|d|+g+2) \\
\quad \text { if } g \neq 0,|d| \leq g \text { and } d \equiv g(\bmod 2) ; \\
\text { (iv) }(0,2,|d|+g+1) \\
\quad \text { if } g \neq 0,|d|<g \text { and } d \neq \equiv(\bmod 2) \text { except }(|d|, g)=(0,1) \text {; } \\
\text { (v) }(0,|d|-g+1,2 g+2) \\
\quad \text { if } g \neq 0,|d| \geq g \text {. }
\end{array}\right.
$$

Figure 2(a) with $\ell=|d|+1$ and $\ell=2$ shows $(c, i, n)$-minimal contours of the cases (i) and (ii) respectively. Figure $2(b)$ with $(j, n)=(0,|d|+g+2),(j, n)=(1,|d|+$ $g+1)$ and $(j, n)=(|d|-g, 2 g+2)$ shows $(c, i, n)$-minimal contours of the cases (iii), (iv) and (v) respectively.

(2) Let $h: F_{g} \rightarrow S^{2}$ be a stable map of modulo two degree $d_{2}$. Then, $\gamma(h)$ is $(c, i, n)$ minimal if and only if the triple $(c, i, n)$ for $\gamma(h)$ is one of the items below:

$$
(c, i, n)= \begin{cases}(1,1,(g+5) / 2) & \text { if } d_{2}=1 \text { and } g \text { is odd; } \\ (0,1,(g+6) / 2) & \text { if } d_{2}=1 \text { and } g \text { is even; } \\ (1,1,(g+7) / 2) & \text { if } d_{2}=0 \text { and } g \text { is odd; } \\ (0,1,(g+8) / 2) & \text { if } d_{2}=0 \text { and } g \text { is even, and } g / 2 \text { is odd } \\ (0,1,(g+4) / 2) & \text { if } d_{2}=0 \text { and } g \text { is even, and } g / 2 \text { is even. }\end{cases}
$$

Theorem 1.2 shows the following corollary.

COROLlary 1.3. Let $f: \Sigma_{g} \rightarrow S^{2}$ be a degree d stable map whose contour is $(c, i, n)$ minimal. Then: 


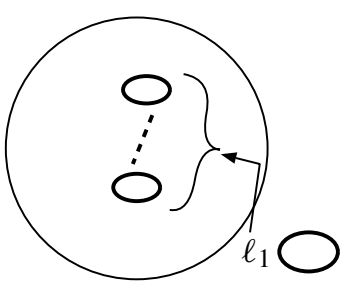

(a)

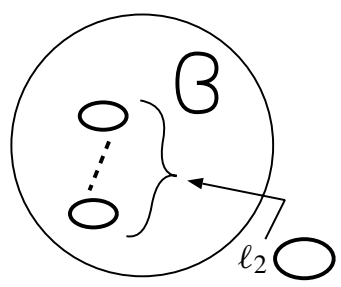

(b)

FIgURE 3. The $(n, c, i)$-minimal contours of $C^{\infty}$ maps $M \rightarrow S^{2}$.

(1) the number $n(f)$ is an even number;

(2) the number $i(f)$ and $\left(\chi\left(\Sigma_{g}\right) / 2\right)+|d|$ have the same parity, where $\chi\left(\Sigma_{g}\right)$ denotes the Euler characteristic of $\Sigma_{g}$.

Note that there is a stable map $\Sigma_{g} \rightarrow S^{2}$ that has an odd number of nodes.

Yamamoto [8] determined the minimal number of fold curve components of fold maps $\Sigma_{g} \rightarrow \Sigma_{h}$. Theorem 1.2 determines the minimal number of nodes among fold maps $\Sigma_{g} \rightarrow S^{2}$ such that the number of fold curve components is minimal.

The following theorem concerning an $(n, c, i)$-minimal contour is the second result of this paper.

\section{THEOREM 1.4.}

(1) Let $f: \Sigma_{g} \rightarrow S^{2}$ be a stable map of degree $d$. Then, $\gamma(f)$ is an $(n, c, i)$-minimal contour if and only if the triple $(n, c, i)$ for $\gamma(f)$ is

$$
(n, c, i)=(0,0,|d|+g+1) .
$$

Figure 3(a) with $\ell_{1}=|d|+g+1$ shows an (n, c, i)-minimal contour of $f$.

(2) Let $h: F_{g} \rightarrow S^{2}$ be a stable map of modulo two degree one. Then, $\gamma(h)$ is an $(n, c, i)$ minimal contour if and only if the triple $(n, c, i)$ for $\gamma(h)$ is

$$
(n, c, i)= \begin{cases}(\mathrm{i})(0,0,(g+4) / 2) & \text { if } g \text { is even; } \\ (\mathrm{ii})(0,1,(g+3) / 2) & \text { if } g \text { is odd } .\end{cases}
$$

Figure 3(a) with $\ell_{1}=(g+4) / 2$ and Figure $3(b)$ with $\ell_{2}=(g+1) / 2$ show $(n, c, i)$ minimal contours of the cases $(i)$ and (ii) respectively.

The following theorem concerning an $(i, n, c)$-minimal contour is the third result of this paper.

THEOREM 1.5.

(1) Let $f: \Sigma_{g} \rightarrow S^{2}$ be a stable map of degree $d$. Then, $\gamma(f)$ is an $(i, n, c)$-minimal contour if and only if the triple $(i, n, c)$ for $\gamma(f)$ is one of the items below:

$$
(i, n, c)= \begin{cases}\text { (i) }(1,0,0) & \text { if } d=g=0 \\ \text { (ii) }(1,0,2(g+2)) & \text { if } d=0 \\ \text { (iii) }(1,0,2(|d|+g)) & \text { otherwise. }\end{cases}
$$




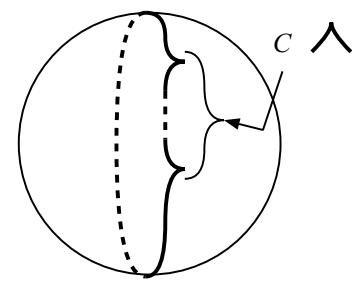

FiguRE 4. The $(i, n, c)$-minimal contour of $C^{\infty}$ maps $M \rightarrow S^{2}$.

Figure 4 with $c=0, c=2(g+2)$ and $c=2(|d|+g)$ shows $(i, n, c)$-minimal contours of the cases (i), (ii) and (iii) respectively.

(2) Let $h: F_{g} \rightarrow S^{2}$ be a stable map of modulo two degree $d_{2}$. Then, $\gamma(h)$ is an $(i, n, c)$ minimal contour if and only the triple $(i, n, c)$ for $\gamma(h)$ is

$$
(i, n, c)=\left(1,0,-2 d_{2}+g+4\right) .
$$

Figure 4 with $c=\left(-2 d_{2}+g+4\right)$ shows an $(i, n, c)$-minimal contour of $h$.

This paper is organized as follows. In Section 2, we prepare some notions concerning the apparent contour of a stable map between surfaces. In Section 3, Theorem 1.2 is proved. In Section 4, Theorem 1.4 is proved. In Section 5, Theorem 1.5 is proved.

Throughout this paper, all surfaces are connected and of class $C^{\infty}$, and all maps are of class $C^{\infty}$. The symbols deg and $\operatorname{deg}_{2}$ denote the mapping degree and the modulo two mapping degree respectively. For a topological space $X$, the symbol $\chi(X)$ denotes the Euler characteristic of $X$.

\section{Preparation}

In the following, we prepare some notions concerning the apparent contour of a stable map of a closed surface into the sphere.

Let $M$ be a closed surface and $\varphi: M \rightarrow S^{2}$ a stable map with singular points. Let $S(\varphi)=S_{1} \cup \cdots \cup S_{\ell}$ be the decomposition of $S(\varphi)$ into the connected components and set $\gamma_{i}=\varphi\left(S_{i}\right)(i=1, \ldots, \ell)$. Note that $\gamma(\varphi)=\gamma_{1} \cup \ldots \cup \gamma_{\ell}$. Denote by $n_{1}(\varphi)$ the sum of the number of self-intersection points of $\gamma_{i}(i=1, \ldots, \ell)$ and $n_{2}(\varphi)$ the sum of the number of points $\gamma_{i} \cap \gamma_{j}$ for all $i$ and $j$ with $i \neq j$. Note that $n_{2}(\varphi)$ is an even number and that $n(\varphi)=n_{1}(\varphi)+n_{2}(\varphi)$. Let $m(\varphi)$ be the smallest number of elements in the set $\varphi^{-1}(y)$, where $y \in S^{2}$ runs over all regular values of $\varphi$. Fix a regular value $\infty$ such that $\varphi^{-1}(\infty)$ consists of $m(\varphi)$ points. For each $\gamma_{i}$, denote by $U_{i}$ the component of $S^{2} \backslash \gamma_{i}$ that contains $\infty$. Note that $\partial U_{i} \subset \gamma_{i}$.

Orient $\gamma_{i}$ so that, at each fold point image, the surface is 'folded to the left'. More precisely, for a point $y \in \gamma_{i}$ that is not a cusp or a node of $\gamma_{i}$, choose a normal vector $v$ of $\gamma_{i}$ at $y$ such that $\varphi^{-1}\left(y^{\prime}\right)$ contains more elements than $\varphi^{-1}(y)$, where $y^{\prime}$ is a regular value of $\varphi$ close to $y$ in the direction of $v$. Let $\tau$ be a tangent vector of $\gamma_{i}$ at $y$ with respect to the above orientation of $\gamma_{i}$. Then, orient $S^{2}$ by the ordered pair $(\tau, v)$. It is easy to see that this gives a well-defined orientation of $S^{2}$. 


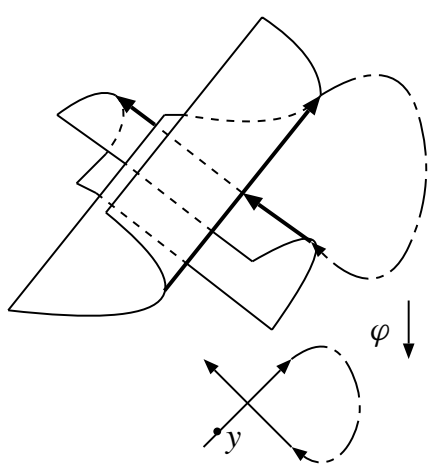

A positive node

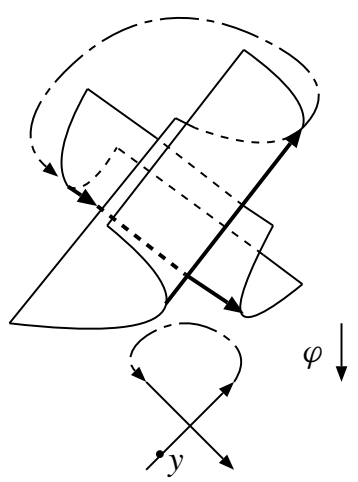

A negative node

FIGURE 5. A positive node and a negative node.

Definition 2.1. A point $y \in \partial U_{i} \backslash\{$ cusps, nodes $\}$ is said to be positive if the normal orientation $v$ at $y$ points toward $U_{i}$; otherwise, it is said to be negative.

A component $\gamma_{i}$ is said to be positive if all points of $\partial U_{i} \backslash$ \{cusps, nodes\} are positive; otherwise, $\gamma_{i}$ is said to be negative. The numbers of positive and negative components are denoted by $i^{+}$and $i^{-}$respectively. Note that there is at least one negative component unless $S(\varphi)=\emptyset$.

Definition 2.2. A point $y \in \partial U_{i} \backslash$ \{cusps, nodes\} is called an admissible starting point if

(1) $y$ is a positive point of a positive component $\gamma_{i}$ or

(2) $y$ is a negative point of a negative component $\gamma_{i}$.

Note that, for each $i$, there always exists an admissible starting point in $\gamma_{i}$.

Definition 2.3. Let $y \in \gamma_{i}$ be an admissible starting point. Suppose that $Q \in \gamma_{i}$ is a node, and let $\alpha:[0,1] \rightarrow \gamma_{i}$ be a parameterization that is consistent with the orientation and is singular only when the image is a cusp such that $\alpha^{-1}(y)=\{0,1\}$. Then, there are two numbers $0<t_{1}<t_{2}<1$ satisfying $\alpha\left(t_{1}\right)=\alpha\left(t_{2}\right)=Q$.

We say that $Q$ is positive if the orientation of $S^{2}$ at $Q$ defined by the ordered pair $\left(\alpha^{\prime}\left(t_{1}\right), \alpha^{\prime}\left(t_{2}\right)\right)$ coincides with that of $S^{2}$ at $Q$; we say that $Q$ is negative, otherwise. See Figure 5 for details.

The numbers of positive and negative nodes on $\gamma_{i}$ are denoted by $N_{i}^{+}$and $N_{i}^{-}$ respectively. The definition of a positive (or negative) node of $\gamma_{i}$ depends on the choice of an admissible starting point $y$. However, it is known that the algebraic number $N_{i}^{+}-N_{i}^{-}$does not depend on the choice of $y$; see [9] for details. Thus, the algebraic number

$$
N^{+}-N^{-}=\sum_{i=1}^{k}\left(N_{i}^{+}-N_{i}^{-}\right)
$$

is well defined. Note that nodes arising from $\gamma_{i} \cap \gamma_{j}(i \neq j)$ play no role in the computation: $n_{1}(\varphi)=N^{+}+N^{-}$. 


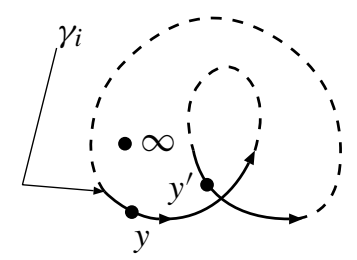

FIGURE 6. Proof of Lemma 2.6.

Then, we have the following formula in [2].

Proposition 2.4. [2] For a stable map $\varphi: M \rightarrow S^{2}$ of a closed surface of genus $g$, we have

$$
g=\varepsilon(M)\left[\left(N^{+}-N^{-}\right)+\frac{c(\varphi)}{2}+\left(1+i^{+}-i^{-}\right)-m(\varphi)\right],
$$

where $\varepsilon(M)$ is equal to 1 if $M$ is orientable and 2 if $M$ is not orientable.

In the following, we assume that $\gamma_{i} \cap \gamma_{j}=\emptyset$ for all $i \neq j$. Denote by $U_{\infty} \subset S^{2} \backslash \gamma(\varphi)$ the component that contains $\infty$. Denote by $\gamma_{1}$ the component of $\gamma(\varphi)$ that contains $\partial U_{\infty}$. Note that $\gamma_{1}$ is a negative component of $\varphi$.

LEMMA 2.5. If $\gamma_{1}$ has a node, then it has a negative node.

Proof. Let us go along $\gamma_{1}$ starting from an admissible starting point $y$, following the canonical orientation of $\gamma_{1}$. When we pass through a positive node of $\gamma_{1}$ for the first time, the number of points in the inverse image decreases by two; see Figure 5 for details. This is a contradiction.

LEMMA 2.6. If a positive component $\gamma_{i}$ has a node, then it has a positive node.

Proof. Let us go along $\gamma_{i}$ starting from an admissible starting point $y$, following the canonical orientation of $\gamma_{i}$. If the first node that we encounter is a negative node (see Figure 5), then the point $y^{\prime} \in \gamma_{i}$ close to the node as in Figure 6 is in $\partial U_{i}$ and $y^{\prime}$ is a negative point. This is a contradiction.

COROLLARY 2.7. If the number of negative components of $\gamma(\varphi)$ is equal to one and $\gamma(\varphi)$ has a node, then it has a negative node.

Proof. We may assume that $\gamma_{1}$ has no node, and that $\gamma_{i}$ is a positive component that has a node and is adjacent to the same component of $S^{2} \backslash \gamma(\varphi)$ as $\gamma_{1}$. Then, by the proof of Lemma 2.6, there is a point $q \in S^{2} \backslash \gamma(\varphi)$ that is close to the node and whose inverse image consists of $m(\varphi)-2$ points (see Figure 7). This is a contradiction.

\section{Proof of Theorem 1.2}

For a $C^{\infty}$ map $\Sigma_{g} \rightarrow S^{2}$ of degree $d$, by chaining the orientation of $\Sigma_{g}$, we obtain a $C^{\infty}$ map $\Sigma_{g} \rightarrow S^{2}$ of degree $-d$. In the following, we assume that $d \geq 0$. 


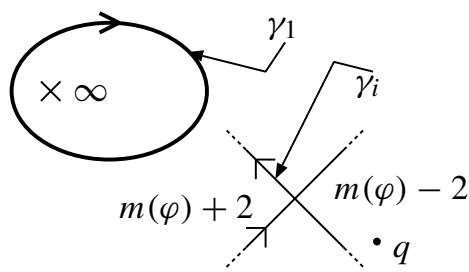

FIGURE 7. Proof of Corollary 2.7.

We remark that the notion of an essential contour of a $C^{\infty}$ map $M \rightarrow N$ was introduced in [5]. Let $\varphi_{0}: M \rightarrow N$ be a $C^{\infty}$ map between surfaces and $\varphi: M \rightarrow N$ a stable map. The contour $\gamma(\varphi)$ is called an essential contour of $\varphi_{0}$ if the triple $(i(\varphi), c(\varphi), n(\varphi))$ is the smallest with respect to the lexicographic order among the stable maps homotopic to $\varphi_{0}$. The following theorem was obtained in [2].

\section{THEOREM 3.1.}

(1) For each $d \geq 0$, the triple ( $i, c, n)$ for an essential contour of a $C^{\infty}$ map $\Sigma_{g} \rightarrow S^{2}$ of degree $d$ is one of the items below:

$$
(i, c, n)= \begin{cases}(1,2 d, 0) & \text { if } g=0 \\ (1,0, d+g+2) & \text { if } g \neq 0, d \leq g \text { and } d \equiv g(\bmod 2) \\ (1,2, d+g+1) & \text { if } g \neq 0, d<g \text { and } d \neq \equiv(\bmod 2) \\ (1,2(d-g), 2 g+2) & \text { if } g \neq 0, d \geq g\end{cases}
$$

(2) The triple $(i, c, n)$ for an essential contour of a $C^{\infty}$ map $F_{g} \rightarrow S^{2}$ of modulo two degree $d_{2}$ is one of the items below:

$$
(i, c, n)= \begin{cases}(1,1,(g+5) / 2) & \text { if } d_{2}=1 \text { and } g \text { is odd; } \\ (1,0,(g+6) / 2) & \text { if } d_{2}=1 \text { and } g \text { is even; } \\ (1,1,(g+7) / 2) & \text { if } d_{2}=0 \text { and } g \text { is odd; } \\ (1,0,(g+8) / 2) & \text { if } d_{2}=0 \text { and } g \text { is even, and } g / 2 \text { is odd } \\ (1,0,(g+4) / 2) & \text { if } d_{2}=0 \text { and } g \text { is even, and } g / 2 \text { is even. }\end{cases}
$$

Note that, if an essential contour of a $C^{\infty}$ map $M \rightarrow S^{2}$ has no cusps or exactly one cusp, then the essential contour is also a $(c, i, n)$-minimal contour. Thus, Theorem 1.2(1) with the case (iii) and 1.2(2) have already been proved.

Let us prove Theorem 1.2(1) except the case (iii).

We prepare some stable maps $\Sigma_{g} \rightarrow S^{2}$ which we employ in the proof of Theorem 1.2. Figure 8 shows the following proposition for a stable map $f: \Sigma_{g} \rightarrow S^{2}$ with singular points.

PROPOSITION 3.2.

(1) By attaching a handle vertically to $f$ (Figure $8(a)$ ), we obtain a stable map $f_{1}: \Sigma_{g+1} \rightarrow S^{2}$ whose quadruple $(i, c, n$, deg) is equal to $(i(f)+1, c(f)$, $n(f), \operatorname{deg} f)$. 

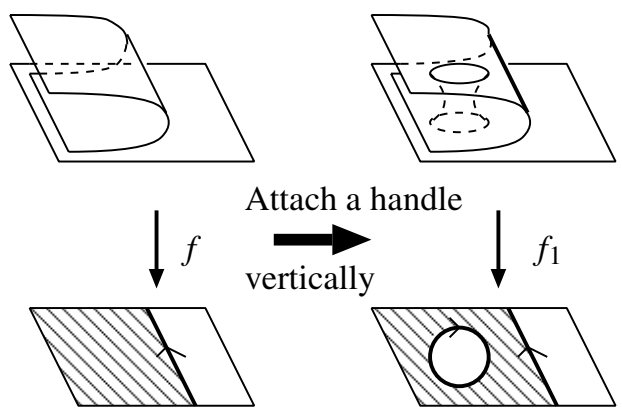

(a)

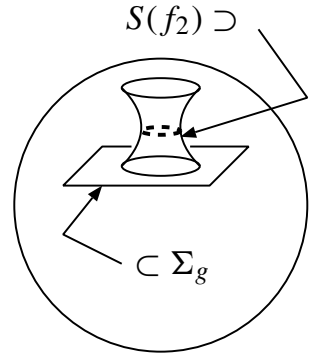

(b)

FIGURE 8. Proof of Proposition 8.

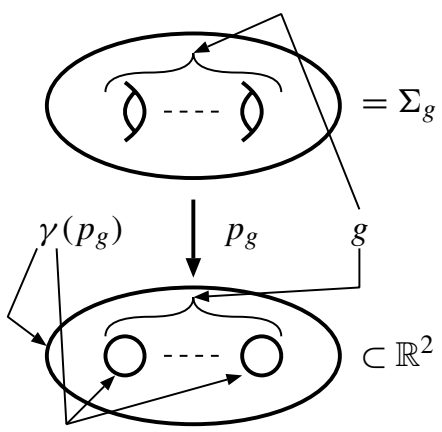

FIGURE 9. A stable map $p_{g}: \Sigma_{g} \rightarrow \mathbb{R}^{2}$.

(2) By attaching a cover vertically to $f$ (Figure $8(b)$ ), we obtain a stable map $f_{2}: \Sigma_{g} \rightarrow S^{2}$ whose quadruple $(i, c, n, \operatorname{deg})$ is equal to $(i(f)+1, c(f), n(f), \operatorname{deg} f+1)$.

Define a degree zero stable map $f_{0,1}^{\prime}: T^{2} \rightarrow S^{2}$ by $f_{0,1}^{\prime}=\iota \circ p_{1}$ where $p_{1}$ is defined by Figure 9 with $g=1$ and $\iota$ is the inclusion $\mathbb{R}^{2} \hookrightarrow \mathbb{R}^{2} \cup\{\infty\}=S^{2}$.

For each pair $(d, g) \neq(0,1)$ satisfying $d<g$ and $d \not \equiv g(\bmod 2)$, there is a degree $d$ stable map $f_{d, g-1}: \Sigma_{g-1} \rightarrow S^{2}$ whose contour is in Figure 2(b) with $(j, n)=(0, d+g+1)$. Then, by attaching a handle vertically (Proposition 3.2(1)) to $f_{d, g-1}$, we obtain a degree $d$ stable map $f_{d, g}^{\prime}: \Sigma_{g} \rightarrow S^{2}$ whose contour is in Figure 2(b) with $(j, n)=(1, d+g+1)$.

For each pair $(d, g)$ satisfying $d \geq g \neq 0$, by attaching $d-g$ covers vertically (Proposition 3.2(2)) to a degree $g$ stable map $f_{g, g}: \Sigma_{g} \rightarrow S^{2}$ whose contour is in Figure 2(b) with $(j, n)=(0,2 g+2)$, we obtain a degree $d$ stable map $f_{d, g}^{\prime}: \Sigma_{g} \rightarrow S^{2}$ whose contour is in Figure 2(b) with $(j, n)=(d-g, 2 g+2)$. On the other hand, by attaching covers vertically (Proposition 3.2(2)) to the degree one stable map $\psi_{1,0}: S^{2} \rightarrow S^{2}$ defined by Figure 10 with $g=0$, we obtain a degree $d \geq 1$ stable map $f_{d, 0}^{\prime}: S^{2} \rightarrow S^{2}$ whose contour is in Figure 2(a) with $\ell=d+1$.

Define $f_{0,0}^{\prime}: S^{2} \rightarrow S^{2}$ by $f_{0,0}^{\prime}=\iota \circ p_{0}$ where $p_{0}$ is defined by Figure 9 with $g=0$, and $\iota: \mathbb{R}^{2} \hookrightarrow S^{2}$ is the inclusion. The triple $(c, i, n)$ for $\gamma\left(f_{0,0}^{\prime}\right)$ is equal to $(0,1,0)$. 


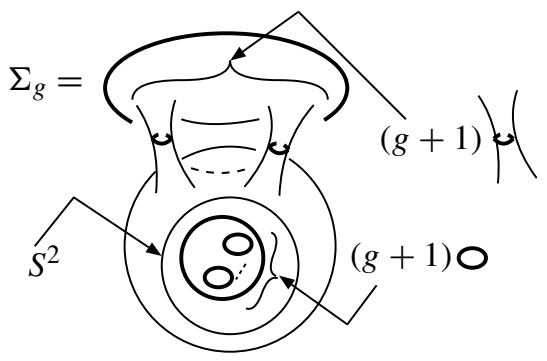

FIGURE 10. A stable map $\psi_{1, g}: \Sigma_{g} \rightarrow S^{2}$.

Thus, we obtain a degree $d$ stable map $f_{d, g}^{\prime}: \Sigma_{g} \rightarrow S^{2}$ whose triple $(c, i, n)$ is equal to the items below:

$$
(c, i, n)= \begin{cases}(0, d+1,0) & \text { if } g=0 ; \\ (0,2,0) & \text { if }(d, g)=(0,1) ; \\ (0,2, d+g+1) & \text { if } g \neq 0, d<g \text { and } d \neq \equiv(\bmod 2) \\ (0, d-g+1,2 g+2) & \text { if } g \neq 0, d \geq g .\end{cases}
$$

Proof of Theorem 1.2(1) except the case (iii). By Proposition 1.1(1), a $(c, i, n)$-minimal contour of a $C^{\infty}$ map $\Sigma_{g} \rightarrow S^{2}$ of degree $d$ consists of at least two components if $g \neq 0$, $d<g$ and $d \not \equiv g(\bmod 2)$. Thus, the contour $\gamma\left(f_{0,1}^{\prime}\right)$ is $(c, i, n)$-minimal and the case (ii) is proved.

Let us prove Theorem 1.2(1) with (iv). Let $f: \Sigma_{g} \rightarrow S^{2}$ be a degree $d$ fold map whose contour consists of two components. We divide this case into the following two parts (1) and (2).

(1) The case of $n_{2}(f)=0$.

(1-1) When $\left(i^{+}, i^{-}\right)$for $\gamma(f)$ is equal to $(1,1)$, then by $(2.1)$, we have

$$
d+g-1 \leq N^{+}-N^{-} .
$$

Therefore we have

$$
n_{1}(f) \geq d+g-1+2 N^{-} .
$$

By (3.1), we have $n_{1}(f)>0$. By Corollary 2.7, we have

$$
n_{1}(f) \geq d+g-1+2 N^{-} \geq d+g+1 .
$$

(1-2) When $\left(i^{+}, i^{-}\right)$for $\gamma(f)$ is equal to $(0,2)$, then, by (2.1), we have

$$
d+g+1 \leq N^{+}-N^{-} .
$$

Therefore we have

$$
n_{1}(f) \geq d+g+1+2 N^{-} \geq d+g+1
$$


(2) The case of $n_{2}(f) \neq 0$.

When $\left(i^{+}, i^{-}\right)$for $\gamma(f)$ is equal to $(2-\lambda, \lambda)(\lambda=1,2)$, then by (2.1), we have $d+g+2 \lambda-3 \leq N^{+}-N^{-}$. Therefore we have

$$
n_{1}(f) \geq d+g+2 \lambda-3+2 N^{-} \geq d+g-1 .
$$

Thus,

$$
n(f)=n_{1}(f)+n_{2}(f) \geq d+g-1+2=d+g+1 .
$$

These show that $\gamma\left(f_{d, g}^{\prime}\right)$ is $(c, i, n)$-minimal if $g \neq 0, d<g$ and $d \not \equiv g(\bmod 2)$ except $(d, g)=(0,1)$. Thus, the case (iv) is proved.

On the other hand, there is the following lemma.

LEMMA 3.3. Let $f: \Sigma_{g} \rightarrow S^{2}$ be a degree d stable map whose contour consists of $\mu$ components $(\mu \neq 0)$. If $|d| \geq g$ and $\mu<|d|-g+1$, then $\gamma(f)$ has at least

$$
2(|d|-g-\mu+1)
$$

cusps.

Proof. Apply the following result of Quine [6]. For a stable map $\varphi: M \rightarrow N$ between oriented surfaces, we have

$$
\chi(M)-2 \chi\left(M_{-}\right)+\sum_{q_{k}: \text { cusp of } f} \operatorname{sign}\left(q_{k}\right)=(\operatorname{deg} \varphi) \chi(N),
$$

where $M_{-}$denotes the closure of the set of regular points $x$ such that the differential $d f_{x}$ reverses the orientation, and $\operatorname{sign}\left(q_{k}\right)(= \pm 1)$ is the $\operatorname{sign}$ of a cusp $q_{k}$ of $f$. See [6] for details.

In our situation, we have:

$$
\sum_{q_{k}: \text { cusp of } f} \operatorname{sign}\left(q_{k}\right)=2\left(d+g-1+\chi\left(\left(\Sigma_{g}\right)_{-}\right)\right) .
$$

Since $\left(\Sigma_{g}\right)_{-}$has $\mu$ boundary components, we have

$$
2-2 g-\mu \leq \chi\left(\left(\Sigma_{g}\right)_{-}\right) \leq \mu .
$$

Thus, we have

$$
2(d-g-\mu+1) \leq \sum_{q_{k}: \text { cusp of } f} \operatorname{sign}\left(q_{k}\right) \leq 2(d+g+\mu-1) .
$$

Then, the result follows immediately.

By Lemma 3.3, the fold curve of a degree $d$ fold map $\Sigma_{g} \rightarrow S^{2}(d \geq g)$ consists of at least $d-g+1$ components. Thus, the contour $\gamma\left(f_{d, 0}^{\prime}\right)$ is $(c, i, n)$-minimal and the case (i) is proved.

By combining similar arguments as the above and the proof of the case (iv), we can show that the contour $\gamma\left(f_{d, g}^{\prime}\right)$ is $(c, i, n)$-minimal for each pair $(d, g)$ satisfying $g \neq 0, d \geq g$. We omit the proof here. Thus, the case (v) is proved.

Therefore, we have completed the proof of Theorem 1.2. 


\section{Proof of Theorem 1.4}

As well as the proof of Theorem 1.2(1), assume $d \geq 0$.

We prepare some stable maps $M \rightarrow S^{2}$ which we shall employ in the proof of Theorem 1.4.

Figure 10 defines a degree one stable map $\psi_{1, g}: \Sigma_{g} \rightarrow S^{2}$ whose contour is in Figure 3(a) with $\ell_{1}=g+2$ for each $g \geq 0$. By attaching covers vertically (Proposition 3.2(2)) to $\psi_{1, g}$ inductively, we obtain a degree $d$ stable map $\psi_{d, g}: \Sigma_{g} \rightarrow S^{2}$ whose contour is in Figure 3(a) with $\ell_{1}=d+g+1$ for each $d \geq 1$ and $g \geq 0$. On the other hand, for each $g \geq 0$, a map $\psi_{0, g}: \Sigma_{g} \rightarrow S^{2}$ that is defined by $\psi_{0, g}=\iota \circ p_{g}$ is a degree zero stable map, where $p_{g}$ is in Figure 9 and $\iota$ is the inclusion $\mathbb{R}^{2} \hookrightarrow S^{2}$. The contour $\gamma\left(\psi_{0, g}\right)$ is in Figure 3(a) with $\ell_{1}=g+1$. Thus, for each $d \geq 0$ and $g \geq 0$, we obtain a degree $d$ stable map $\psi_{d, g}: \Sigma_{g} \rightarrow S^{2}$ whose triple $(n, c, i)$ is

$$
(n, c, i)=(0,0, d+g+1) .
$$

There is a modulo two degree one stable map $\phi_{1,2}: F_{2} \rightarrow S^{2}$ whose contour is in Figure 3(a) with $\ell_{1}=3$. By attaching handles vertically to $\phi_{1,2}$ inductively, we obtain a modulo two degree one stable map $\phi_{1, g}: F_{g} \rightarrow S^{2}$ whose contour is in Figure 3(a) with $\ell_{1}=(g+4) / 2$ for each even number $g$.

For the case that $g$ is an odd number, we prepare the stable map below.

Proposition 4.1. There is a modulo two degree one stable map $\phi_{1,1}: \mathbb{R} P^{2} \rightarrow S^{2}$ whose contour is in Figure $3(b)$ with $\ell_{2}=1$.

Proof. To construct the stable map, divide $\mathbb{R} P^{2}$ into three parts; a Möbius band $\widetilde{M}$, an annulus $A$, and a disk $D$. Define $A \rightarrow \mathbb{R}^{3}$ and $\widetilde{M} \rightarrow \mathbb{R}^{3}$ by Figure 11 (a) and (b) respectively. Note that each map has a Whitney umbrella point. By sticking the boundary of $\widetilde{M}$ to the boundary of $A$ along the solid line in Figure 11(a) and (b), we obtain a stable map $\phi: M \rightarrow \mathbb{R}^{3}$ that has two Whitney umbrella points, where $M$ is homeomorphic to a Möbius band. Then, we define $\left.\phi_{1,1}\right|_{M}: M \rightarrow S^{2}$ by $\iota \circ \pi \circ \phi$, where $\pi: \mathbb{R}^{3} \rightarrow \mathbb{R}^{2}$ is the projection and $\iota: \mathbb{R}^{2} \hookrightarrow S^{2}$ is the inclusion. Finally, by sticking the boundary of $M$ to the boundary of $D$ along the dotted line (Figure 12), we obtain the desired map $\phi_{1,1}: \mathbb{R} P^{2} \rightarrow S^{2}$.

By attaching handles vertically (Proposition 3.2) to $\phi_{1,1}$, we obtain a modulo two degree one stable map $\phi_{1, g}: F_{g} \rightarrow S^{2}$ whose contour is in Figure 3(b) with $\ell_{2}=(g+1) / 2$ for each odd number $g$.

Thus, for each $g \geq 1$, we have a modulo two degree one stable map $\phi_{1, g}: F_{g} \rightarrow S^{2}$ whose triple $(n, c, i)$ is

$$
(n, c, i)= \begin{cases}(0,0,(g+4) / 2) & \text { if } g \text { is an even number, } \\ (0,1,(g+3) / 2) & \text { if } g \text { is an odd number. }\end{cases}
$$

Proof of Theorem 1.4(1). By (2.1), for a stable map $f: \Sigma_{g} \rightarrow S^{2}$ whose contour has no node and no cusp, we have

$$
d+g+1 \leq i(f) .
$$

Therefore, the contour $\gamma\left(\psi_{d, g}\right)$ is $(n, c, i)$-minimal for each $d$ and $g$. Thus, Theorem 1.4(1) has been proved. 


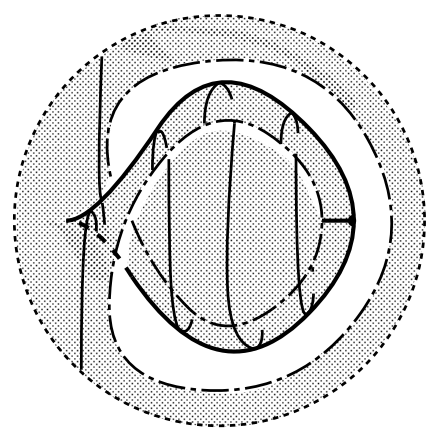

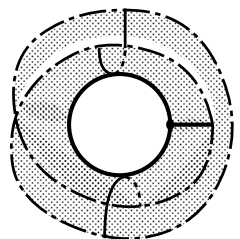

(b)

(a)

FIGURE 11.

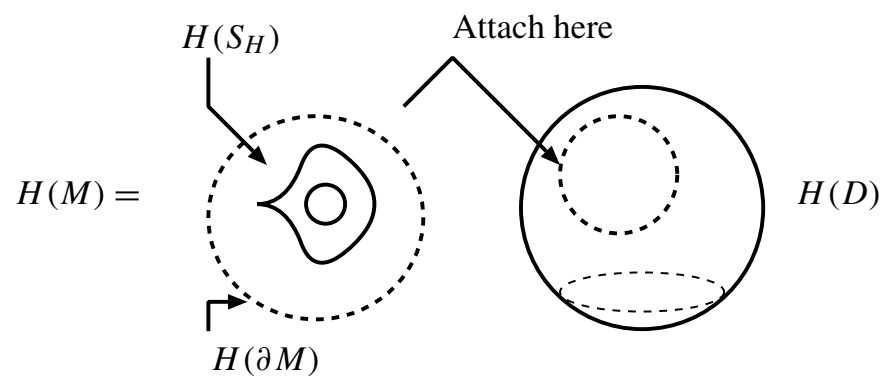

(a)

(b)

FIGURE 12.

For each $g$, we can prove that the contour $\gamma\left(\phi_{1, g}\right)$ is $(n, c, i)$-minimal in a similar way as Theorem 1.4(1). Thus, Theorem 1.4(2) is proved. We omit the proof here.

Finally in this section, we pose the following problem.

Problem 4.2. Let us study an $(n, c, i)$-minimal contour of a $C^{\infty}$ map $F_{g} \rightarrow S^{2}$ of modulo two degree zero.

\section{Proof of Theorem 1.5}

We prepare some stable maps $M \rightarrow S^{2}$ which we shall employ in the proof of Theorem 1.5. Figure 13 shows the following proposition for a stable map $f: \Sigma_{g} \rightarrow S^{2}$ with singular points.

Proposition 5.1.

(1) By attaching a handle horizontally to $f$ (Figure 13(a)), we obtain a stable map $f_{3}: \Sigma_{g+1} \rightarrow S^{2}$ whose quadruple $(i, c, n$, deg) is equal to $(i(f), c(f)+2$, $n(f), \operatorname{deg} f)$. 

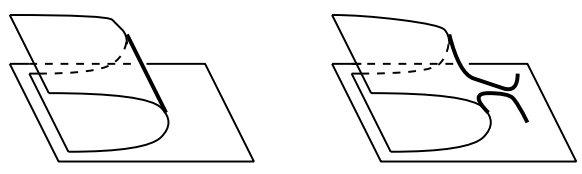

Attach a handle

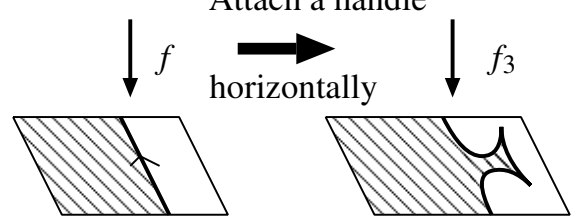

(a)

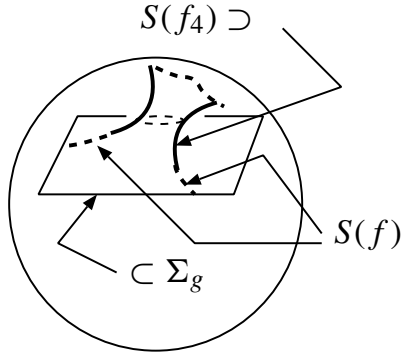

(b)

FIGURE 13.

(2) By attaching a cover horizontally (Figure 13(b)) to $f$, we obtain a stable map $f_{4}: \Sigma_{g} \rightarrow S^{2}$ whose quadruple $(i, c, n, \operatorname{deg})$ is equal to $(i(f), c(f)+2$, $n(f), \operatorname{deg} f+1)$.

Define a degree zero stable map $u_{0,0}: S^{2} \rightarrow S^{2}$ by $\iota \circ p_{0}$ where $p_{0}$ is in Figure 9 with $g=0$ and $\iota: \mathbb{R}^{2} \hookrightarrow S^{2}$ is the inclusion.

By making a pleat (Figure 14) to $\mathrm{id}_{S^{2}}$, we obtain a degree one stable map $u_{1,0}: S^{2} \rightarrow S^{2}$ whose contour is in Figure 4(a) with $c=2$, where $\mathrm{id}_{S^{2}}$ is the identity map on $S^{2}$. By attaching handles or covers horizontally (Proposition 5.1(1) and (2)) to $u_{1,0}$ inductively, we obtain a degree $d$ stable map $u_{d, g}: \Sigma_{g} \rightarrow S^{2}$ whose contour is in Figure 4(a) with $c=2(d+g)$ for each $d \geq 1$ and $g \geq 0$. By attaching a cover that is turned over to $u_{1,1}$, we obtain a degree zero stable map $u_{0,1}: T^{2} \rightarrow S^{2}$ whose contour is in Figure 4(a) with $c=6$. By attaching handles horizontally (Proposition 3.2(1)) inductively to $u_{0,1}$, we obtain a degree zero stable map $u_{0, g}: \Sigma_{g} \rightarrow S^{2}$ whose contour is in Figure 4(a) with $c=2(g+2)$. Thus, for each $g$, we have a degree $d$ stable map $u_{d, g}: \Sigma_{g} \rightarrow S^{2}$ whose triple $(i, n, c)$ is

$$
(i, n, c)= \begin{cases}(1,0,0) & \text { if } d=g=0 \\ (1,0,2(g+2)) & \text { if } d=0 \\ (1,0,2(d+g)) & \text { otherwise. }\end{cases}
$$

We constructed modulo two degree one stable maps $h_{1,1}: \mathbb{R} P^{2} \rightarrow S^{2}$ and $h_{1,2}: F_{2} \rightarrow S^{2}$ whose contours are in Figure 4 with $c=3$ and $c=4$ in [2] respectively. By applying a similar argument as $u_{d, g}$ to $h_{1,1}$ and $h_{1,2}$, we obtain a modulo two degree $d_{2}$ stable map $\eta_{d_{2}, g}: F_{g} \rightarrow S^{2}$ whose contour is in Figure 4 with $c=-2 d_{2}+g+4$ for each $d_{2}$ and $g$.

Proof of Theorem 1.5(1). The contour $\gamma\left(u_{0,0}\right)$ is trivially $(i, n, c)$-minimal. Thus, the case (i) is proved.

Note that there is no stable map $f: \Sigma_{g} \rightarrow S^{2}$ with $m(f)=0$ whose contour is irreducible, and whose contours have no node and have cusps by the geometrical reason of a cusp. By combining this reason and (2.1), for the contour of a degree zero stable map $f: \Sigma_{g} \rightarrow S^{2}$ whose contour is irreducible and has no nodes, we have

$$
2(g+2) \leq 2(g+m(f))=c .
$$



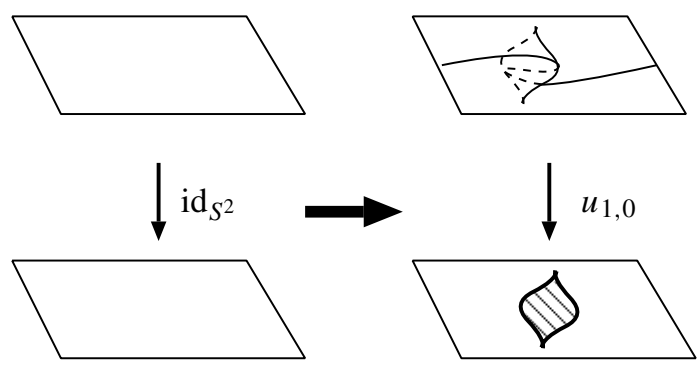

FIGURE 14. Pleat.

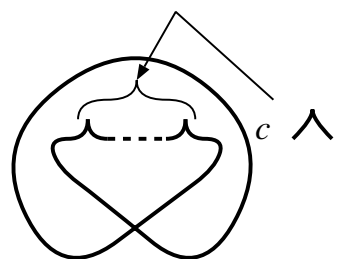

(a)

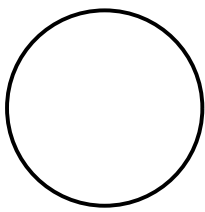

(b)

FiguRE 15 . The $(i, n, c)$-minimal contours of stable maps $M \rightarrow \mathbb{R}^{2}$.

Thus, the contour $\gamma\left(u_{0, g}\right)$ is $(i, n, c)$-minimal for each $g>0$, and the case (ii) is proved.

By (2.1), for the contour of a degree $d$ stable map $f: \Sigma_{g} \rightarrow S^{2}$ whose contour is irreducible and has no nodes, we have

$$
2(d+g) \leq 2(g+m(f))=c .
$$

Thus, the contour $\gamma\left(u_{d, g}\right)$ is $(i, n, c)$-minimal for each $d \geq 1$ and $g \geq 0$, and the case (iii) is proved.

Therefore, the proof of Theorem 1.5(1) has been completed.

We can prove that $\gamma\left(\eta_{d_{2}, g}\right)$ is $(i, n, c)$-minimal by a similar argument as the case (1), for each $d_{2}$ and $g$. Thus, Theorem 1.5(2) is proved. We omit the proof here.

The following theorem is also obtained by similar arguments.

\section{THEOREM 5.2.}

(1) Let $f: \Sigma_{g} \rightarrow \mathbb{R}^{2}$ be a stable map. Then, the contour $\gamma(f)$ is $(i, n, c)$-minimal if and only if the triple $(i, n, c)$ for $\gamma(f)$ is

$$
(i, n, c)= \begin{cases}\text { (i) }(1,1,2 g+2) & \text { if } g \geq 1 ; \\ \text { (ii) }(1,0,0) & \text { if } g=0 .\end{cases}
$$

Figure 15(a) with $c=2 g+2$ and 15(b) show $(i, n, c)$-minimal contours of the cases $(i)$ and (ii) respectively. 
(2) Let $h: F_{g} \rightarrow \mathbb{R}^{2}$ be a stable map. The contour $\gamma(h)$ is $(i, n, c)$-minimal if and only if the triple $(i, n, c)$ for $\gamma(h)$ is

$$
(i, n, c)=(1,1, g+2) .
$$

Figure 15(a) with $\ell=g+2$ shows an $(i, n, c)$-minimal contour of $h$.

Acknowledgements. The author would like to express his gratitude to Osamu Saeki for helpful comments and constant encouragement. The author also thanks the editor and the referee for useful comments that improved this paper. The author also expresses special thanks to Akiko Neriugawa for useful advice on English grammar and for encouraging support.

\title{
REFERENCES
}

[1] S. Demoto. Stable maps between 2-spheres with a connected fold curve. Hiroshima Math. J. 35(1) (2005), 93-113.

[2] A. Kamenosono and T. Yamamoto. The minimal numbers of singularities of stable maps between surfaces. Topology Appl. 156 (2009), 2390-2405.

[3] J. Milnor. Topology from the differentiable viewpoint. Based on notes by David W. Weaver. University Press of Virginia, Charlottesville, VA, 1965.

[4] R. Pignoni. Minimal arrangements of singularities for apparent contours. C. R. Acad. Sci. Paris Sér. I Math. 313(12) (1991), 873-878.

[5] R. Pignoni. Projections of surfaces with a connected fold curve. Topology Appl. 49 (1993), 55-74.

[6] J. R. Quine. A global theorem for singularities of maps between oriented 2-manifolds. Trans. Amer. Math. Soc. 236 (1978), 307-314.

[7] R. Thom. Les singularités des applications différentiables (French). Ann. Inst. Fourier, Grenoble 6 (19551956), 43-87.

[8] M. Yamamoto. The number of singular set components of fold maps between oriented surfaces. Houston J. Math. To appear.

[9] H. Whitney. On regular families of curves. Bull. Amer. Math. Soc. 47 (1941), 145-147.

[10] H. Whitney. On singularities of mappings of euclidean spaces. I. Mappings of the plane into the plane. Ann. of Math. (2) 62 (1955), 374-410.

\author{
Takahiro Yamamoto \\ Faculty of Engineering \\ Kyushu Sangyo University \\ 3-1 Matsukadai 2-chome \\ Higashi-ku \\ Fukuoka 813-8503 \\ Japan \\ (E-mail:yama.t@ip.kyusan-u.ac.jp)
}

